EXTENDED REPORT

\title{
Level of acceptability of EULAR recommendations for the management of knee osteoarthritis by practitioners in different European countries
}

\section{B Mazières, N Schmidely, H J Hauselmann, E Martin-Mola, U Serni, A A Verbruggen, M Le Bars}

See end of article for authors' affiliations

.....................

Correspondence to: Professor B Mazières, Department of Rheumatology, University Hospital of Rangueil, 1 Avenue Jean-Poulhès, 31059 Toulouse Cedex 9, France; mazieres@cict.fr

Accepted 30 January 2005 Published Online First 11 February 2005

\begin{abstract}
Objective: To evaluate the level of acceptability of the EULAR recommendations for the management of knee osteoarthritis (KOA) in practice.

Methods: A questionnaire was sent to general practitioners, rheumatologists, rehabilitators, and orthopaedic surgeons in five European countries (France, Spain, Belgium, Switzerland, Italy). Practitioners were asked to give their opinion on the 10 EULAR recommendations and on 23 treatment modes for KOA. Practitioners' opinions were compared with those of the expert task force involved in the development of these recommendations.

Results: The overall response rate was $10.4 \%$ (4204 replies). Results were similar across countries and specialties. Of the 23 treatment modes proposed, only joint lavage and intra-articular (IA) corticosteroid injections were more strongly recommended by the expert task force than by the responders as a whole, while the opposite was true for spa therapy. Principal component analysis showed: (1) some practitioners preferred "hard line" treatments (surgery, IA injections, or non-steroidal anti-inflammatory drugs (NSAIDs)); (2) there was a difference between those prescribing pharmacological (paracetamol) or nonpharmacological measures with low iatrogenicity (exercises, sticks, education), and those prescribing less well validated treatments closer to "alternative" medicine; (3) each specialist tended to advocate modes that they were most familiar with: rheumatologists were more likely to recommend IA injections and NSAIDs; orthopaedic surgeons, surgical procedures; rehabilitators, education and all non-pharmacological modes; general practitioners, spa therapy and opioids.

Conclusions: A multidisciplinary approach is optimal in the management of this chronic disease with its variable course.
\end{abstract}

\section{METHODS}

A questionnaire was sent to general practitioners, rheumatologists, rehabilitators, and orthopaedic surgeons in five
European countries-Belgium, France, Italy, Spain, and Switzerland-between February and April 2001.

In all countries the questionnaire was sent by the scientific societies of each specialty involved, to all their members. However, in Switzerland, a general mailing was undertaken to all general practitioners, rheumatologists (including rehabilitators), and orthopaedic surgeons, using a national database. In Italy, only rheumatologists were involved.

The two questions addressed in this paper are:

- What is your overall opinion about the usefulness of each of the 23 treatment modes of the European recommendations on the treatment of knee osteoarthritis (using a $100 \mathrm{~mm}$ visual analogue scale (VAS), ranging from 0 "I do not recommend its use" to 100 "I recommend its use")?

- What is your overall opinion on each of the 10 European recommendations on the treatment of knee osteoarthritis (using a $100 \mathrm{~mm}$ visual analogue scale ranging from 0 "I totally disagree with this recommendation" to 100 "I totally agree")?

Completed questionnaires were collected and data were recorded and analysed, using SAS 8.2 software for PC (SAS Institute Inc, Cary, North Carolina, USA), as follows. Descriptive analyses (means, standard deviations, medians, and quartiles) and comparisons between practitioners' and

Abbreviations: EULAR, European League Against Rheumatism; KOA knee osteoarthritis; PCA, principal components analysis; VAS, visual analogue scale 
Table 1 Final recommendations for the management of knee osteoarthritis based on the opinion of the experts (from reference 1)

1 The treatment of knee OA should be tailored to the individual patient, taking into account factors such as age, comorbidity, and the presence of inflammation

2 Optimal management of knee OA requires a combination of pharmacological and non-pharmacological treatment modes

3 Intra-articular injection of a long acting steroid is indicated for acute exacerbation of knee pain, especially if accompanied by effusion

4 There is evidence that SYSADOA (glucosamine sulphate, chondroitin sulphate, diacerein, and hyaluronic acid) may possess structure modifying properties, but more studies using standardised methods are required

5 Hyaluronic acid and other SYSADOA are probably effective in knee OA, but the size effect is relatively small, suitable patients are not well defined, and pharmacoeconomic aspects of this treatment are not well established

6 Non-pharmacological treatment of knee OA should include regular education, exercise, appliances (sticks, insoles), and weight reduction

7 Exercises, especially those directed towards increasing strength of quadriceps and/or preserving normal mobility of the knee, are strongly recommended

8 Paracetamol is the oral analgesic to try first and, if successful, is the preferred long term oral analgesic

9 NSAIDs (oral and topical) should be considered in patients (with effusion) unresponsive to paracetamol

10 Joint replacement has to be considered in cases of refractory pain associated with disability and radiological deterioration

NSAID, non-steroidal anti-inflammatory drug; OA, osteoarthritis; SYSADOA, symptomatic slow acting drugs for osteoarthritis.

experts' opinions were carried out on the whole survey population, and by country and specialty. Comparisons of the mean scores between countries (whatever the specialty), and between specialties (whatever the country), were made using an overall analysis of variance (Fisher's test). Multivariate descriptive analysis using principal components analysis (PCA) was employed to summarise high dimensional data into fewer dimensions. ${ }^{5}$ PCA aimed to examine relations among all the answers provided by practitioners. This statistical analysis "reduces" data to the pertinent components with respect to variability and identifies correlations between therapeutic modes; these can determine "medical practices" or "general opinions" within the sample of the survey participants. In these newly calculated scales, the country effect and the specialty effect on medical practices and general opinion can be examined.

Missing answers were considered as missing data in the analyses. In the multivariate analysis we used only fully completed questionnaires.

\section{RESULTS}

More than 40000 questionnaires were sent out, to nearly 30000 general practitioners, 3500 rheumatologists, more than 2000 rehabilitators, and 4900 orthopaedic surgeons in five European countries. As the questionnaires were sent by the scientific society to their members only, not all physicians were represented. This explains the variability in the percentage of practitioners across countries and specialties (table 2).

In all, 4204 completed questionnaires were returned, corresponding to $10.4 \%$ of the practitioners contacted. This percentage varied across countries (from $6.5 \%$ (Spain) to $18 \%$ (Italy), and across specialty (from $7.4 \%$ of general practitioners to $22.9 \%$ of rheumatologists) (table 2).

Nine hundred and sixty four questionnaires $(23 \%$ of responses) contained one or more incomplete items and each item was completed in at least $91 \%$ of the returned questionnaires. PCA were conducted on the 3240 fully completed questionnaires.

The age distribution of practitioners was almost identical in France, Belgium, and Switzerland (table 2). For France, the age distribution of physicians was based on the national statistics, as an indicator of sample representativeness. In all countries, between one quarter and one fifth of practitioners were in the 30 to 40 year age group. Spain had the highest percentage of practitioners in the 41 to 50 year age group.
Data from Italy were insufficient and the percentage of given answers was too small to be analysed. Fifty two per cent of practitioners answering this question (94.6\% response rate) were in private practice, $29 \%$ in a hospital, and $19 \%$ had a mixed mode of practice.

\section{Opinions on treatment modes}

The overall results of the survey were compared to the EULAR task force experts' opinions ${ }^{1}$ (table 3 ). The results were globally similar. Only three treatment modes were judged differently by experts and survey participants: joint lavage and intra-articular (IA) steroid injections were more strongly recommended by the experts (mean (SD): 55 (28) $\mathrm{mm}$ and 71 (27) $\mathrm{mm}$, respectively) than by the survey participants as a whole (30 (26) $\mathrm{mm}$ and 46 (27) $\mathrm{mm}$ ). Conversely, the survey participants were more in favour of spa therapy (49 (29) mm) than the experts of the task force $(28(22) \mathrm{mm})$.

Some differences were observed between countries: practitioners in Belgium were less in favour of weight reduction (mean (SD): $44(37) \mathrm{mm}$ ) and spa therapy (36 (29) $\mathrm{mm}$ ) compared with the global average response $(69$ (31) $\mathrm{mm}$ and $49(29) \mathrm{mm}$ ) (table 4). The following treatment modes were more often recommended in some countries compared with the average: osteotomy $(64(28) \mathrm{mm})$ and joint lavage (40 (28) $\mathrm{mm}$ ) in France; use of insoles (58 (27) mm and 61 (29) $\mathrm{mm}$ ) in Switzerland and Italy; and topical creams (57 (28) $\mathrm{mm}$ ) in Switzerland. All differences were statistically significant though not clinically relevant, except for vitamins, where opinions were the same across countries (14 (18) mm in the whole survey).

Some differences were observed between specialties (table 5): total knee replacement and osteotomy were more often recommended by orthopaedic surgeons (mean (SD): 86 (16) $\mathrm{mm}$ and 72 (25) $\mathrm{mm}$, respectively), while exercise (66 (27) $\mathrm{mm})$ and insoles (30 (28) $\mathrm{mm})$ were less often recommended compared with other specialties. General practitioners were more in favour of opioids (30 (26) $\mathrm{mm}$ ) and less likely to prescribe hyaluronic acid $(28(23) \mathrm{mm})$, while rheumatologists were strong advocates of IA steroid administration (60 (24) $\mathrm{mm}$ ).

To summarise the large amount of information collected, four principal components were identified in the PCA multivariate analysis:

- Component 1 (18.3\% of total variability): this opposed the practitioners who gave high scores to all items (compared with the mean values of the total survey) to those who 
Table 2 Details of the questionnaire mailing, by country, and by specialty, and the demographics of the physicians involved

\begin{tabular}{|c|c|c|c|c|c|c|}
\hline & Belgium & France & Italy & Spain & Switzerland & Total \\
\hline $\begin{array}{l}\text { Number of inhabitants (millions } \\
\text { in 2001) }\end{array}$ & 10.3 & 59.7 & 57.8 & 40.1 & 7.3 & 175.2 \\
\hline \multicolumn{7}{|c|}{ Specialties (number ( $\%$ of the total number of specialists in the country)) } \\
\hline GPs & $4757(31.3 \%)$ & $6070(8.3 \%)$ & 40 & $12000(50 \%)$ & $7147(100 \%)$ & 29984 \\
\hline Rheumatologists & $199(58.2 \%)$ & $905(34.6 \%)$ & $921(100 \%)$ & $1070(100 \%)$ & $352 *(100 \%)$ & 3447 \\
\hline Rehabilitators & $367(74.7 \%)$ & $444(38.1 \%)$ & 39 & $1300(100 \%)$ & - & 2150 \\
\hline Orthopaedic surgeons & $732(67.7 \%)$ & $1535(49 \%)$ & - & $2100(100 \%)$ & $555(100 \%)$ & 4922 \\
\hline \multicolumn{7}{|l|}{ Questionnaires ( $\mathrm{n}$ ) } \\
\hline Number sent & 6055 & 8924 & 1000 & 16470 & 8054 & 40503 \\
\hline Number returned (\% sent) & $1067(17.6 \%)$ & $1174(13.2 \%)$ & $180(18.0 \%)$ & $1071(6.5 \%)$ & $712(8.8 \%)$ & $4204(10.4 \%)$ \\
\hline \multicolumn{7}{|c|}{ Age group of physicians (years) (\% in sample/\% in national database) } \\
\hline $30-40$ & $25 / 25.4 \dagger$ & $21 / 17.6 \ddagger$ & 36 & 25 & 18 & \\
\hline $41-50$ & $37 / 31.0$ & $41 / 45.0^{+}$ & 52 & 57 & 43 & \\
\hline $51-60$ & $25 / 21.3$ & $30 / 30.9$ & 10 & 12 & 32 & \\
\hline$>60$ & $13 / 22.3$ & $8 / 6.0$ & 2 & 5 & 6 & \\
\hline
\end{tabular}

*In the Swiss health system, it is not possible to differentiate between rheumatologists and rehabilitators.

†National Statistics for Belgium: http://www.statbel.fgov.be/pub/d3/p363y2000_fr.pdf

$\ddagger$ National Statistics for France: DREES, 2001 (D Sicart - Les médecins: estimates for 1 January 2001 to 28 October 2001): http://www.sante.gouv.fr/ GP, general practitioner.

gave low scores to all items. Belgium among countries and orthopaedic surgeons among specialties scored all items lower than the mean values of the total survey.

- Component 2 (9.3\% of total variability): this corresponded to consistency in practitioners' preference for knee replacement, osteotomy, joint lavage, IA steroids, arthroscopic debridement, IA hyaluronic acid, and NSAIDs. French practitioners were more likely to recommend these "hard line" treatments than Spanish or Italian. Similarly, orthopaedic surgeons were more likely to recommend these treatment methods than general practitioners.

- Component 3 (7.9\% of total variability): this opposed recommendations for exercise, sticks, patient education, and paracetamol (which were positively related) to recommendations for vitamins, mineral salts, and hormone replacement therapy. Italians, Spanish, and Swiss physicians were more likely to recommend the first group than the second group, while Belgian physicians were more likely to do the reverse. Similarly, orthopaedic surgeons were more likely to recommend the first group than the second group, while general practitioners were more likely to recommend the second group.

- Component 4 (5.5\% of variability): this opposed practitioners' age group to the recommendations for opioids, NSAIDs, and IA steroids. These treatment modes were recommended less often by older physicians and also less often in Belgium and France. On the other hand, rheumatologists, who were younger, were more likely to

Table 3 Assessment of treatment modes in the whole survey compared with the experts' opinion, ${ }^{1}$ expressed in $\mathrm{mm}$ on a visual analogue scale, where $0=$ "I do not recommend its use", and $100=$ "I do recommend its use"

\begin{tabular}{llll}
\hline & & \multicolumn{2}{l}{ Survey (total) } \\
\cline { 3 - 4 } & Experts' opinion & No of responses & Mean value (mm) \\
\hline Exercise & $82(15)$ & 4069 & $76(23)$ \\
TKR & $81(33)$ & 4038 & $70(24)$ \\
Paracetamol & $78(22)$ & 4195 & $76(23)$ \\
NSAIDs & $77(24)$ & 4196 & $66(22)$ \\
Education & $77(25)$ & 4055 & $75(24)$ \\
IA steroids & $71(27)$ & 4196 & $46(27)$ \\
Diet & $69(29)$ & 4061 & $69(31)$ \\
Sticks & $60(32)$ & 4063 & $67(24)$ \\
Lavage & $55(28)$ & 3959 & $30(26)$ \\
Insoles & $54(34)$ & 4020 & $47(30)$ \\
SYSADOA & $50(27)$ & 3975 & $47(27)$ \\
Topics & $50(29)$ & 4010 & $44(29)$ \\
Hyaluronic acid & $48(24)$ & 3902 & $35(26)$ \\
Osteotomy & $42(31)$ & 4004 & $49(29)$ \\
Opioids & $40(21)$ & 4186 & $27(25)$ \\
Patellar tape & $35(28)$ & 3824 & $23(22)$ \\
Arthroscopic debridement & $32(28)$ & 3962 & $34(26)$ \\
Psychotropic drugs & $28(23)$ & 4035 & $17(18)$ \\
Spa therapy & $28(22)$ & 4049 & $49(29)$ \\
Telephone contact & $27(26)$ & 3882 & $30(27)$ \\
Vitamins & $19(22)$ & 4015 & $14(18)$ \\
HRT & $15(19)$ & 4001 & $12(16)$ \\
Minerals & $14(15)$ & 4010 & $19(22)$ \\
\hline
\end{tabular}

Values are mean (SD) or $\mathrm{n}$.

HRT, hormone replacement therapy; IA, intra-articular; NSAIDs, non-steroidal anti-inflammatory drugs; SYSADOA, symptomatic slow acting drugs for osteoarthritis; TKR, total knee replacement. 


\begin{tabular}{|c|c|c|c|c|c|c|c|}
\hline & Survey (total) & Belgium & France & Italy & Spain & Switzerland & p Value* \\
\hline No of questionnaires & 4204 & 1067 & 1174 & 180 & 1071 & 712 & \\
\hline Exercise & $76(23)$ & $72(25)$ & $69(26)$ & 83 (17) & $81(18)$ & $82(20)$ & $<0.0001$ \\
\hline TKR & $70(24)$ & $69(23)$ & $76(22)$ & $55(29)$ & $67(25)$ & $72(23)$ & $<0.0001$ \\
\hline Paracetamol & $76(23)$ & $74(23)$ & $79(21)$ & $66(27)$ & $76(22)$ & $75(25)$ & $<0.0001$ \\
\hline Education & $75(24)$ & $71(25)$ & $74(25)$ & $87(16)$ & $79(21)$ & $73(24)$ & $<0.0001$ \\
\hline NSAIDs & $66(22)$ & $62(23)$ & $68(22)$ & $68(22)$ & $64(20)$ & $74(20)$ & $<0.0001$ \\
\hline IA steroids & $46(27)$ & $44(26)$ & $49(29)$ & $50(25)$ & $41(25)$ & $52(26)$ & $<0.0001$ \\
\hline Diet & $69(31)$ & $44(37)$ & $80(22)$ & $86(17)$ & $77(21)$ & $74(26)$ & $<0.0001$ \\
\hline Sticks & $67(24)$ & $62(26)$ & $68(24)$ & $65(25)$ & $70(21)$ & $68(24)$ & $<0.0001$ \\
\hline Lavage & $30(26)$ & $22(24)$ & $40(28)$ & $33(27)$ & $28(24)$ & $28(25)$ & $<0.0001$ \\
\hline Insoles & $47(30)$ & $42(30)$ & $43(31)$ & $61(29)$ & $46(28)$ & $58(27)$ & $<0.0001$ \\
\hline SYSADOA & $47(27)$ & $44(27)$ & $51(28)$ & $56(26)$ & $42(24)$ & $50(27)$ & $<0.0001$ \\
\hline Topics & $44(29)$ & $40(28)$ & $42(29)$ & $43(30)$ & $42(27)$ & $57(28)$ & $<0.0001$ \\
\hline Hyaluronic acid & $35(26)$ & $29(25)$ & $38(26)$ & $58(26)$ & $35(24)$ & $33(27)$ & $<0.0001$ \\
\hline Osteotomy & $49(29)$ & $39(28)$ & $64(28)$ & $40(27)$ & $46(26)$ & $50(27)$ & $<0.0001$ \\
\hline Opioids & $27(25)$ & $20(23)$ & $30(28)$ & $14(18)$ & 27 (22) & $33(27)$ & $<0.0001$ \\
\hline Patellar tape & $23(22)$ & $23(21)$ & $22(22)$ & $26(22)$ & $22(20)$ & $26(24)$ & 0.00004 \\
\hline Arthroscopic debridement & $34(26)$ & $33(26)$ & $32(27)$ & $36(26)$ & $33(25)$ & $37(26)$ & 0.00007 \\
\hline Psychotropic drugs & $17(18)$ & $14(17)$ & 16 (18) & $15(20)$ & $21(18)$ & $18(18)$ & $<0.0001$ \\
\hline Spa therapy & $49(29)$ & $36(29)$ & $50(28)$ & $60(27)$ & $63(25)$ & $45(28)$ & $<0.0001$ \\
\hline Telephone contact & $30(27)$ & $23(25)$ & $30(27)$ & $44(31)$ & $34(28)$ & $29(27)$ & $<0.0001$ \\
\hline Vitamins & $14(18)$ & $13(17)$ & $14(19)$ & $13(16)$ & $14(17)$ & $14(20)$ & 0.11193 \\
\hline HRT & $12(16)$ & $10(16)$ & $16(20)$ & $9(12)$ & $13(15)$ & $8(12)$ & $<0.0001$ \\
\hline Minerals & $19(22)$ & $17(21)$ & $20(24)$ & $15(18)$ & $21(22)$ & $17(21)$ & 0.00001 \\
\hline
\end{tabular}

Values are mean (SD).

*p Values for Fisher's test of country effect (analysis of variance).

HRT, hormone replacement therapy; IA, intra-articular; NSAIDs, non-steroidal anti-inflammatory drugs; SYSADOA, symptomatic slow acting drugs for osteoarthritis; TKR, total knee replacement.

recommend these methods than orthopaedic surgeons, who were older.

These four components accounted for $41 \%$ of total variability. All further identified components accounted for too little variability to provide useful comparisons.

\section{Opinion on the EULAR recommendations}

Overall, the physicians were strongly in favour of the recommendations (table 6). The scores ranged from 93 (11) $\mathrm{mm}$ for tailored treatment (recommendation 1) to 76 (27) $\mathrm{mm}$ for IA steroid injections (recommendation 3). There were very few differences between countries (table 6) and

Table 5 Assessment of treatment modes in the whole survey and by specialty, expressed in mm on a visual analogue scale, where $0=$ "I do not recommend its use", and $100=$ "I do recommend its use"

\begin{tabular}{|c|c|c|c|c|c|c|}
\hline & Survey (total) & GPs & Rheumatologists & Rehabilitators & $\begin{array}{l}\text { Orthopaedic } \\
\text { surgeons }\end{array}$ & p Value* \\
\hline No of questionnaires & 4204 & 2213 & 790 & 260 & 845 & \\
\hline Exercise & $76(23)$ & $77(21)$ & $80(20)$ & $79(22)$ & $66(27)$ & $<0.0001$ \\
\hline TKR & $70(24)$ & $64(24)$ & $69(25)$ & $74(21)$ & $86(16)$ & $<0.0001$ \\
\hline Paracetamol & $76(23)$ & $79(21)$ & 77 (22) & $73(24)$ & $68(26)$ & $<0.0001$ \\
\hline Education & $75(24)$ & $75(23)$ & $81(21)$ & $83(19)$ & $67(27)$ & $<0.0001$ \\
\hline NSAIDs & $66(22)$ & $65(22)$ & $67(21)$ & 65 (22) & $71(22)$ & $<0.0001$ \\
\hline IA steroids & $46(27)$ & $42(25)$ & $60(24)$ & 47 (27) & 44 (29) & $<0.0001$ \\
\hline Diet & $69(31)$ & $67(32)$ & $76(26)$ & $66(32)$ & $68(31)$ & $<0.0001$ \\
\hline Sticks & $67(24)$ & $67(24)$ & $66(23)$ & $72(23)$ & $65(25)$ & 0.0018 \\
\hline Lavage & $30(26)$ & $23(23)$ & $38(26)$ & $30(26)$ & $40(28)$ & $<0.0001$ \\
\hline Insoles & $47(30)$ & $53(28)$ & $50(30)$ & $45(28)$ & $30(28)$ & $<0.0001$ \\
\hline SYSADOA & 47 (27) & $46(27)$ & $53(27)$ & $49(27)$ & $42(26)$ & $<0.0001$ \\
\hline Topics & $44(29)$ & $49(28)$ & $42(29)$ & $44(30)$ & $32(27)$ & $<0.0001$ \\
\hline Hyaluronic acid & $35(26)$ & $28(23)$ & $48(27)$ & $40(25)$ & $40(26)$ & $<0.0001$ \\
\hline Osteotomy & 49 (29) & $42(26)$ & 45 (27) & $52(29)$ & 72 (25) & $<0.0001$ \\
\hline Opioids & $27(25)$ & $30(26)$ & $24(24)$ & $22(24)$ & $21(23)$ & $<0.0001$ \\
\hline Patellar tape & $23(22)$ & $26(21)$ & $23(22)$ & $22(22)$ & $17(20)$ & $<0.0001$ \\
\hline Arthroscopic debridement & $34(26)$ & $33(24)$ & $29(24)$ & $27(24)$ & $42(29)$ & $<0.0001$ \\
\hline Psychotropic drugs & 17 (18) & $20(19)$ & $14(16)$ & $13(16)$ & $14(16)$ & $<0.0001$ \\
\hline Spa therapy & $49(29)$ & $49(29)$ & $52(27)$ & $56(29)$ & $45(29)$ & $<0.0001$ \\
\hline Telephone contact & $30(27)$ & $32(27)$ & $33(28)$ & $25(27)$ & $22(24)$ & $<0.0001$ \\
\hline Vitamins & $14(18)$ & $15(20)$ & $11(16)$ & $11(16)$ & $12(16)$ & $<0.0001$ \\
\hline HRT & $12(16)$ & 13 (17) & $10(15)$ & 13 (19) & 10 (15) & $<0.0001$ \\
\hline Minerals & $19(22)$ & $22(24)$ & $13(19)$ & $16(20)$ & $16(19)$ & $<0.0001$ \\
\hline
\end{tabular}

Values are mean $(\mathrm{mm})$.

*p Values for Fisher's test of country effect (analysis of variance).

GP, general practitioner; HRT, hormone replacement therapy; IA, intra-articular; NSAIDs, non-steroidal anti-inflammatory drugs; SYSADOA, symptomatic slow acting drugs for osteoarthritis; TKR, total knee replacement. 
Table 6 Assessment of recommendations in the whole survey and by country, expressed in mm on a visual analogue scale, where $0=$ "I totally disagree with this recommendation", and $100=$ "I totally agree with this recommendation"

\begin{tabular}{|c|c|c|c|c|c|c|c|}
\hline & Survey (total) & Belgium & France & Italy & Spain & Switzerland & p Value* \\
\hline No of questionnaires & 4204 & 1067 & 1174 & 180 & 1071 & 712 & \\
\hline Tailored treatment & $93(11)$ & $92(12)$ & $92(12)$ & $95(10)$ & $94(11)$ & $95(9)$ & $<0.0001$ \\
\hline Combined treatment & 91 (15) & $89(16)$ & 88 (17) & $95(11)$ & 93 (12) & $94(11)$ & $<0.0001$ \\
\hline Paracetamol first & $82(24)$ & $78(25)$ & $86(20)$ & 68 (32) & $85(21)$ & 77 (27) & $<0.0001$ \\
\hline NSAIDs & $83(21)$ & $77(25)$ & 85 (19) & $79(28)$ & 85 (17) & 88 (19) & $<0.0001$ \\
\hline Steroid injection & $76(27)$ & $75(26)$ & $73(30)$ & $88(18)$ & 76 (27) & $82(24)$ & $<0.0001$ \\
\hline SYSADOA/structure & $77(26)$ & 77 (26) & $76(27)$ & $84(22)$ & $76(25)$ & 78 (24) & 0.00082 \\
\hline HA/SYSADOA small effect & $78(25)$ & $79(25)$ & $78(26)$ & 72 (29) & 79 (24) & $78(26)$ & 0.01683 \\
\hline Non-pharmacological treatments & $91(14)$ & 89 (16) & 90 (15) & $94(12)$ & $93(11)$ & 93 (12) & $<0.0001$ \\
\hline Exercises recommended & $88(18)$ & $86(18)$ & 83 (22) & 93 (12) & $92(13)$ & 93 (12) & $<0.0001$ \\
\hline Total knee replacement & $92(14)$ & 91 (14) & $90(15)$ & 93 (12) & $92(13)$ & 93 (12) & 0.00024 \\
\hline
\end{tabular}

Values are mean (SD).

*p Value for Fisher's test of country effect (analysis of variance).

HA, hyaluronic acid; NSAID, non-steroidal anti-inflammatory drug; SYSADOA, symptomatic slow acting drugs for osteoarthritis.

specialties (table 7), other than the observation that rheumatologists were more likely to recommend corticosteroid injections (90 (15) $\mathrm{mm}$ ) than physicians overall (76 (27) $\mathrm{mm})$.

Principal component analysis also identified several pertinent components:

- Component 1 (30\% of total variability): this opposed the practitioners who gave high scores to all items (compared with the mean values of the total survey) to those who gave low scores to all items. Belgium and France among the five countries and orthopaedic surgeons among the specialties scored all items lower than the mean values of the total survey.

- Component 2 (10.5\% of variability): this corresponded to consistency in practitioners' preference for recommendations 8 and 9 referring to paracetamol and NSAIDs (see table 1). Those who agreed with these recommendations tended not to favour recommendations 4 and 5, relating to hyaluronic acid and symptomatic slow acting drugs for osteoarthritis (SYSADOA) (see table 1). Belgian and Italian physicians had a greater preference for hyaluronic acid and SYSADOA, whereas their Swiss, Spanish, and French counterparts were more in favour of NSAIDs and paracetamol. Similarly, orthopaedic surgeons, and to a lesser extent rheumatologists, were in favour of NSAIDs and paracetamol rather than hyaluronic acid and SYSADOA, whereas the reverse was true for general practitioners.
- Component 3 (9.4\% of variability): this opposed recommendations 8 and 9 referring to paracetamol and NSAIDs, as well as age of the practitioners, to recommendations 6 and 7 , relating to non-pharmacological treatments and exercise. Italy, Spain, Belgium, and Switzerland (equal third), followed by France (in descending order) preferred recommendations 8 and 9 . With reference to specialties, orthopaedic surgeons, who were older, preferred NSAIDs and paracetamol, whereas the other specialists, who were younger, were more in favour of non-pharmacological treatments.

- Component $4(9.0 \%$ of the variability): this opposed recommendation 10 referring to joint replacement to recommendation 9, referring to NSAIDs. Spanish and French physicians preferred NSAIDs to joint replacement whereas the opposite was true for the Swiss; the Italian and Belgian physicians were closer to the Swiss position. Not surprisingly, orthopaedic surgeons preferred prostheses and general practitioners tended to choose NSAIDs.

These four components accounted for 59\% of overall variability.

\section{DISCUSSION}

We report the results of a European survey of the views of the various specialists involved in the management of knee osteoarthritis. Overall, they showed a high degree of acceptance of the recommendations by the practitioners

Table 7 Assessment of recommendations in the whole survey and by specialty, expressed in mm on a visual analogue scale, where $0=$ "I totally disagree with this recommendation", and $100=$ "I totally agree with this recommendation"

\begin{tabular}{|c|c|c|c|c|c|c|}
\hline & Survey (total) & GPs & Rheumatologists & Rehabilitators & $\begin{array}{l}\text { Orthopaedic } \\
\text { surgeons }\end{array}$ & p Value* \\
\hline No of questionnaires & 4204 & 2213 & 790 & 260 & 845 & \\
\hline Tailored treatment & $93(11)$ & $93(11)$ & $94(10)$ & $95(8)$ & $93(12)$ & 0.00095 \\
\hline Combined treatment & $91(15)$ & $92(12)$ & $94(11)$ & $93(11)$ & $86(20)$ & $<0.0001$ \\
\hline Paracetamol first & $82(24)$ & $84(21)$ & $81(25)$ & 81 (27) & $75(28)$ & $<0.0001$ \\
\hline NSAIDs & $83(21)$ & 84 (19) & $84(22)$ & $82(25)$ & $80(24)$ & 0.00001 \\
\hline Steroid injection & $76(27)$ & $75(26)$ & $90(15)$ & 79 (25) & $67(33)$ & $<0.0001$ \\
\hline SYSADOA/structure & $77(26)$ & $76(24)$ & $81(25)$ & $82(23)$ & $73(29)$ & $<0.0001$ \\
\hline HA/SYSADOA small effect & $78(25)$ & $78(25)$ & $78(26)$ & 81 (23) & $79(26)$ & 0.15537 \\
\hline Non-pharmacological treatments & $91(14)$ & $92(13)$ & $93(11)$ & $94(11)$ & $88(18)$ & $<0.0001$ \\
\hline Exercises recommended & $88(18)$ & $89(16)$ & $92(13)$ & 91 (17) & $81(24)$ & $<0.0001$ \\
\hline Total knee replacement & $92(14)$ & $90(14)$ & $92(13)$ & 93 (13) & 93 (13) & $<0.0001$ \\
\hline
\end{tabular}

Values are mean (SD).

*p Values for Fisher's test of country effect (analysis of variance).

GP, general practitioner; HA, hyaluronic acid; NSAIDs, non-steroidal anti-inflammatory drugs; SYSADOA, symptomatic slow acting drugs for osteoarthritis. 
and little difference between the opinions of the European practitioners and those of the EULAR task force ${ }^{1}$ concerning the treatments used in KOA. The greatest difference was that the experts were more likely to recommend IA corticosteroid injections and joint lavage than European practitioners, who were more likely to recommend spa therapy.

The overall percentage of physicians who returned a completed questionnaire may seem quite low (10.4\%); however, the absolute number of questionnaires analysed was high (4204), allowing analysis in subgroups, defined by countries or specialties.

We checked the representativeness of the survey by considering France and Belgium as an example. We compared the age of the physicians who replied to the questionnaire with that of the whole population of physicians and found very similar distribution curves, suggesting that the survey sample is representative of the whole population of physicians. In addition, all relevant specialties were contacted (that is, all members of scientific societies involved in the management of osteoarthritis). We also checked the representativeness of the general practitioners involved in the survey in France, as the number might seem very low compared with the total number of GPs. However, we found that our sample was indeed representative of the GPs involved in the management of osteoarthritis.

This survey had some limitations. For example, certain therapeutic modes (lavage, ${ }^{6}$ arthroscopic debridement, ${ }^{7}$ patellar strapping ${ }^{8}$ ) were evidently not clearly understood by some physicians and should have been better defined in the questionnaire.

Also, the overall responses should be interpreted with caution owing to the large number of GPs participating in the survey. To clarify the results, we carried out a principal components analysis. This revealed associations between treatment modes and identified differences between medical practices within the sample of responses. We found the following. First, orthopaedic surgeons, as opposed to general practitioners, were more likely to recommend "hard line" treatments (so called because they are invasive or potentially toxic). The positive association between such treatment modes suggests that physicians who prefer certain types of treatment are likely to prescribe others of a similar type. Second, we also showed differences between two groups of treatment methods: on the one hand, pharmacological treatment (paracetamol) or non-pharmacological measures (exercise, sticks, education) that are reasonably well validated and have low iatrogenicity were most often prescribed by orthopaedic surgeons and less often by general practitioners; on the other hand, treatments that are not so well validated (such as hormonal therapy) or "alternative medicines" (that is, vitamins, mineral salts) were most often prescribed by GPs and less often by orthopaedic surgeons. Finally, the age of the prescribers may play a role in the choice of treatment ${ }^{9}$ as younger physicians were more likely than their older peers to prescribe opioids, IA corticosteroids, and NSAIDs.

Principal components analysis of opinion about the 10 recommendations showed the same trends: orthopaedic surgeons were more in favour of the recommendations on NSAIDs and paracetamol than GPs and less in favour of those concerning symptomatic slow acting drugs for osteoarthritis (SYSADOA) and non-pharmacological modes including exercise, and vice versa. Not surprisingly, orthopaedic surgeons were more likely than GPs to recommend knee replacement than the use of oral NSAIDs, and vice versa. The age of the practitioners also played a role, as orthopaedic surgeons (older) were more in favour of recommendations on paracetamol and NSAIDs than other specialists (younger) and less in favour of those on non-pharmacological modes.
Overall, such analyses suggest that the management of the patient with knee osteoarthritis depends in part on the age, specialty, and country of origin of the practitioner consulted. It was interesting that rheumatologists were more likely to prescribe local injections (of both corticosteroids and hyaluronic acid) and NSAIDs, that orthopaedic surgeons most commonly recommended surgical procedures, that rehabilitators recommended non-pharmacological measures, and that general practitioners preferred spa therapy and the use of opioids. Different specialists are seeing patients at different stages of the disease, which may have a direct impact on their prescriptions.

In the management of a chronic disease with variable and progressive clinical manifestations, it is normal that treatment measures should be graduated. At any given stage of the disease, an optimised strategy should be determined using an evidence based approach, and this should favour a multidisciplinary form of management of this chronic disease. Further studies are needed to establish predictive factors of response to treatment and decision trees allowing a "tailored" treatment.

Although we found high scores of agreement with the recommendations, we do not know the extent to which practitioners are ready to apply all these recommendations in clinical practice. A recent survey conducted in general practice in France allowed such an evaluation. ${ }^{10}$ The major findings were that there was a very low prescription rate of non-pharmacological treatments (ranging from 9\% for exercise to $42 \%$ for joint sparing advice), a high rate of prescription of paracetamol as first line treatment (90\%), a high rate of co-prescription of NSAIDs as first line treatment $(43 \%)$, and a low degree of prescription of corticosteroid injections in case of flare up (22\%). These results reflect different degrees of compliance with the guidelines.

The existing gap between research and practice has been highlighted on several previous occasions, and studiesincluding those involving osteoarthritis guidelines-have been carried out to define effective interventions in osteoarthritis. ${ }^{41}$ As far as physicians are concerned, lectures, distribution of recommendations, and audiovisual materials are of little use, while interactive educational meetings are consistently effective at promoting behavioural changes among health professionals. ${ }^{12}$ To conclude, our findings suggest that a multidisciplinary approach is optimal in the management of this chronic disease with such a variable course.

\section{ACKNOWLEDGEMENTS}

We thank Bristol-Myers Squibb Company for CRF design, data collection, and analysis and mailing costs.

\section{Authors' affiliations}

B Mazières, University Hospital of Rangueil, Toulouse, France

N Scmidely, M Le Bars, Bristol-Myers Squibb Company, Rueil-

Malmaison, France

H J Hauselmann, Centre for Rheumatology and Bone Disease, Zurich, Switzerland

E Martin-Mola, Hospital La Paz, Madrid, Spain

U Serni, Istituto Ortopedico Toscano, Firenze, Italy

A A Verbruggen, Universiteit Ziekenhuis, Gent, Belgium

\section{REFERENCES}

1 Pendleton A, Arden N, Dougados M, Doherty M, Bannwarth B, Bijlsma JWJ, et al. EULAR recommendations for the management of knee osteoarthritis: report of a task force of the Standing Committee for International Clinical Studies including Therapeutic Trials (ESCISIT). Ann Rheum Dis 2000;59:936-44.

2 Agence Nationale d'Accréditation et d'Evaluation en Santé (ANAES). Efficacité des methodes de mise en oeuvre des recommandations médicales. Paris: ANAES, 2000. 
3 Hagemeister J, Schneider CA, Barabas S, Schadt R, Wassmer G, Mager G, et al. Hypertension guidelines and their limitations - the impact of physician's compliance as evaluated by guideline awareness. J Hypertens $2001 ; 19: 2079-86$.

4 Bouvenot J, Gentile S, Ousset S, Tardieu S, Jouve E, Mendizabal H, et al. [Factors influencing the appropriation of clinical guidelines by physicians]. Presse Med 2002;31:1831-5.

5 Cooley WW, Lohnes PR. Multivariate data analysis, vol 1. New York: John Wiley and Sons, 1971

6 Ravaud P, Moulinier L, Giraudeau B, Ayral X, Guerin C, Noel E, et al. Effects of joint lavage and steroid injection in patients with osteoarthritis of the knee: results of a multicenter, randomized, controlled trial. Arthritis Rheum 1998;42:475-82.

7 Fond J, Rodin D, Ahmad S, Nirschl RP. Arthroscopic debridement for the treatment of osteoarthritis of the knee: 2- and 5-year results. Arthroscopy 2002; 18:829-34

8 Cushnaghan J, McCarthy C, Dieppe P. Taping the patella medially: a new treatment for osteoarthritis of the knee joint? BMJ 1994;308:753-5.
9 Hagemeister J, Schneider CA, Barabas S, Schadt R, Wassmer G, Mager G, et al. Hypertension guidelines and their limitations. The impact of physicians' compliance as evaluated by guideline awareness. J Hypertens $2001 ; 19: 2079-86$.

10 Chevalier X, Marre JP, de Butler J, Hercek A. Questionnaire survey of management and prescription of general practitioners in knee osteoarthritis: a comparison with 2000 EULAR recommendations. Clin Exp Rheumatol 2004;22:205-12

11 Ray WA, Stein CM, Byrd V, Shorr R, Pichert JW, Gideon P, et al. Educational program for physicians to reduce use of non-steroidal anti-inflammatory drugs among community-dwelling elderly persons: a randomized controlled trial. Med Care 2001;39:425-35.

12 Bero LA, Grilli R, Grimshaw JM, Harvey E, Oxman AD, Thomson MA. Closing the gap between research and practice: an overview of systematic reviews of interventions to promote the implementation of research findings. BMJ 1998;317:465-8. 